\title{
Where does a cohesive granular heap break?
}

\author{
Lydéric Bocquet, ${ }^{1, *}$ Frédéric Restagno, ${ }^{2,}$ 团 and Élisabeth Charlaix ${ }^{1, \text { 团 }}$ \\ 1 Laboratoire physique de la matière condensée et nanostructures UMR CNRS 5586 \\ Université Lyon I - 43, Bd du 11 Novembre 1918 - 69622 Villeurbanne Cedex - France \\ ${ }^{2}$ Laboratoire de physique des solides - UMR CNRS 8502 - Bat. 510 - Campus universitaire, \\ 91405 Orsay Cedex, France
}

(Dated: November 12, 2018)

\begin{abstract}
In this paper, we consider the effect of cohesion on the stability of a granular heap and compute the maximum angle of stability of the heap as a function of the cohesion. We show that the stability is strongly affected by the dependence of the cohesion on the local pressure. In particular, this dependence is found to determine the localization of the failure plane. While for a constant cohesive force, slip occurs deep inside the heap, surface failure is obtained for a linear variation of the cohesion on the normal stress. Such a transition allows to interpret some recent experimental results on cohesive materials.
\end{abstract}

PACS numbers: 61.43.Gt, 61.43.Gt, 45.70.Cc

\section{INTRODUCTION}

The economic impact of particle processing is enormous. Better methods for the design and synthesis of unit operations involving divided solids have been identified as a critical need, especially for pharmaceutical, agrochemicals and specialty chemicals. One of the important features in industrial processes is to better understand the transition between a static granular medium and the avalanche process. Avalanches in non-cohesive granular media have been extensively investigated 1, 2]. A common characteristic of these studies is that granular motion occurs in a relative thin boundary layer (around ten grains) at the surface [3] independent of the size of the sample. On the other hand, recent experiments have explored the relatively new subject of cohesive granular media such as "humid granular" media. The presence of capillary bridges between the grains generate adhesive forces which strongly affect the stability of the samples [4, 5, 6, 7, 8, 9, 10, 11]. These capillary bridges either originate in small amounts of added fluid, and this situations corresponds to "lightly wet granular media" or either are created by a condensable vapor in the atmosphere 7, 8, 9]. In the latter case the vapor is in chemical equilibrium with the interstitial liquid bridges. These experiments concern the so-called "moist granular media".

As shown by all these experiments, the behavior of humid granular media strongly departs from the dry materials and many new features appear. In a series of experiments on "lightly wet" granular media, Tegzes et al. [12], measured the angle of repose of a granular media (by the draining crater method) as a function of the liquid content and of the size of the system. They observed an increase of the angle of repose with the liquid content.

*Electronic address: lbocquet@lpmcn.univ-lyon1.fr

${ }^{\dagger}$ Electronic address: restagno@lps.u-psud.fr

‡Electronic address: charlaix@lpmcn.univ-lyon1.fr
More precisely, they obtained three regimes: i) at low liquid content, the "granular regime", the avalanche occurs at the surface and the angle of repose does not depend on the size of the system; ii) at intermediate liquid content, the "correlated regime", the avalanche takes place more deeply in the heap, the angle of repose depends on the size of the system; iii) at high liquid content, a kind of "plastic flow" is observed. This various regimes are not accounted for by previous theoretical analysis.

In numerical and analytical modelizations, cohesion is usually taken into account as a constant adhesion force. As an example, Forsyth et al. [13] and Valverde et al. 10] used a constant van der Waals force to interpret their experimental results, Olivi-Tran et al. [14] used a constant capillary force or a constant force due to a solid bridge in particle dynamics (PD) simulations, Albert et al. 44] used a constant capillary force in a geometrical model and Nase et al. [15], Mikami et al. [16] used a constant capillary force in PD simulations.

In this paper we show that the assumption of a constant cohesion is not justified in many practical cases. We then show that the dependence of the cohesion on normal stress, usually omitted in the literature, is a key point in determining the stability of a cohesive granular heap. This effect leads to a dependence of the stability on the system size, as found experimentally. To this end, we shall use a continuum analysis [17] to study the stability of cohesive sandpiles taking into account a non constant cohesive stress in the pile. The paper is divided in two parts. We briefly first show that in a many situations, the adhesion force between the grains depends on the normal stress. Then, in the second part, we show how this relationship affects the stability of a heap and the localization of the slip plane upon failure.

\section{CONTACT FORCES BETWEEN GRAINS}

The adhesion force between two ideal spheres due to solid-solid interactions has been calculated in the case of 
an elastic contact a long time ago [18, 19, 20]:

$$
F_{S-S}=f \pi \gamma R
$$

where $R$ is the sphere radius, $\gamma$ is the solid/intersticial medium surface tension $\left(\gamma=\gamma_{S G}\right.$ if the spheres are in a gas atmosphere, and $\gamma=\gamma_{S L}$ if they are immersed in a liquid), and $f$ is a numerical factor between 1.5 and 2 . The lower value of $f=1.5$ corresponds to the JohnsonKendall-Roberts calculation (JKR) valid when the attractive forces are strong enough to deform the spheres surfaces. The higher value $f=2$ corresponds to the Derjaguin-Muller-Toporov (DMT) calculation, which is valid in the opposite limit of weak attraction and rigid solids. The main hypothesis in obtaining Eq. 1 is that surfaces are ideal. An obvious feature of this result is that adhesion does not depend on the pressure applied on the surfaces before to separate them [21]. However measured values of the adhesion force generally departs from the predicted one [22]. As we briefly discuss in the next section, the effect of surface roughness leads to qualitatively different results, involving a dependence of the adhesion force on the normal load.

\section{A. Pressure depending adhesion between two grains}

Restagno et al. 22 have recently measured the adhesion force between two weakly rough surfaces (a sphere and a plane) using a surface forces apparatus. The "pulloff" force $F_{\text {adh }}$ to separate two surfaces has been found to depend on the a loading normal force, $F$, with a scaling: $F_{\text {adh }} \sim F^{1 / 3}$.

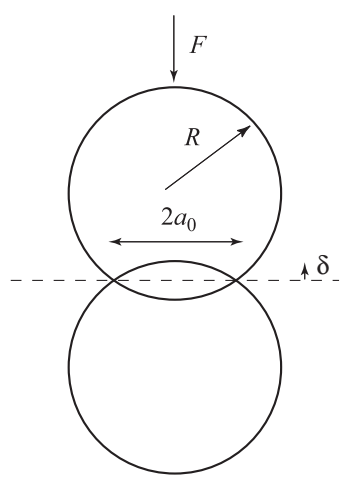

(a)

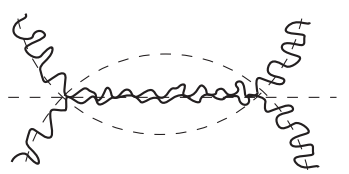

(b)
FIG. 1: (a): Contact between two perfect spheres of radius $R$, Young modulus $E$.

(b): Contact between two rough particules of radius $R$.

The key-point to interpret this result is the roughness of the surfaces as pointed out 50 years ago by MacFarlane and Tabor 23]: the surfaces are in contact only at the tip of their asperities, and the real contact area $A_{\mathrm{r}}$ is much smaller than the apparent contact area $A_{\text {app }}$ (Fig. [1. To obtain the adhesion force they used Eq. 10 with an effective surface tension $\gamma_{\mathrm{eff}}=\gamma A_{\mathrm{r}} / A_{\mathrm{app}}$. On one hand, different ways can be used to calculate $A_{\mathrm{r}}$. The first way is to consider that the pressure in the molecular contact area is so high than it can reach the yield stress of the material [23]. An other way has been proposed by Greenwood 24] considering the statistical distribution of heights of the surface asperities. This two models lead to a simple proportionality between the real contact area $A_{r}$ and the normal load:

$$
A_{\mathrm{r}} \sim F
$$

On the other hand, the calculation of the apparent contact is the elastic contact area calculated by Hertz [25] : $A_{\text {app }} \sim\left(F R / E^{*}\right)^{2 / 3}$, with $E^{*}=E /\left[2\left(1-\nu^{2}\right)\right]$ the reduced Young modulus of the material, defined in terms of the Young modulus $E$ and the Poisson modulus $\nu$ of the material. Gathering results, one obtains eventually $\gamma_{\text {eff }} \sim F^{1 / 3}$, so that Eq. पis compatible with the observed experimental scaling.

In the previous experiments, the cohesion between two rough surfaces due to the direct interaction between the surfaces has been considered. However, adhesion might also originate in the presence of a cohesive "binder" in between the surfaces. In many practical situations, the presence of liquid bridges connecting the grains leads to strong adhesive forces between the grains [4, 5, 6, 7, 8, 9]. When the material is placed in an atmosphere of a condensable vapor [7, [8], the size of the liquid bridge is fixed via chemical equilibrium by the value of external humidity. The size of such bridges is very small, around a few nanometers, and one expects that the liquid bridges will condense only in the regions close to real contact: One expects therefore that $F_{\text {coh }} \sim A_{\text {r }}$, the area of real contact area (see e.g. 7] for a more complete discussion). Since as discussed above in Eq. 2] $A_{\mathrm{r}}$ is proportional to the external load, one gets eventually: $F_{\text {adh }} \sim F$. This picture is however valid when the size $r$ of the bridges is lower than a typical scale of roughness, $\ell_{R}$ : In the opposite case, $r>\ell_{R}$, roughness is not pertinent any more and the capillary force reduces to a contant value $2 \pi \gamma R$, with $R$ the radius of the beads, and $\gamma$ the liquid vapor surface tension.

To sum up, the adhesion force between two surfaces can be written:

$$
F_{\text {adh }} \sim F^{n}
$$

with various expected exponents $n$. For humid granular materials, a transition from from $n=1$ to $n=0$ is expected, depending on the relative size of the capillary bridges (i.e. on the added amount of liquid) compared to roughness : For small amount of liquid, the value $n=1$ is expected, while large amounts of liquid correspond to $n=0$. On the other hand, for dry or liquid saturated materials in which cohesion is expected to originate in direct (e.g. van der Waals) interactions, a value $n=1 / 3$ is predicted.

We now derive the local cohesion stress from the adhesion force. 


\section{B. from adhesion force to local cohesion stress}

The problem of the derivation of the interparticle forces $F$ from the bulk stress $\sigma$ is an old problem. Quintanilla et al. 11] have shown, comparing direct adhesion between particules using an AFM and cohesion stress measured by determining the tensile stress of a material, that adhesion increases with the normal load in the material and that a correlation between the microscopic contact force and a macroscopic stress measurement can be performed with a good precision.

For a system of hard monodisperse spherical particles with a random isotropic packing, a continuum-like picture leads to a simple linear relationship between force $F$ and stress $\sigma[26]$ :

$$
\sigma=\frac{\phi k}{4 \pi R^{2}} F
$$

where $R$ is the radius of the particles, $k$ is the coordination number, which is defined as the average number of contacts per particle, and $\phi$ is the volume fraction. Such a relationship has been widely used in the literature for powders or wet granular media [1, 27]. From Eq. 4] we can deduce the relationship between the adhesion force $F_{\text {adh }}$ and the adhesive stress $c_{\text {adh }}$ in the granular medium:

$$
c_{\text {adh }}=\frac{\phi k}{4 \pi R^{2}} F_{\text {adh }} \sim \frac{F_{\text {adh }}}{R^{2}}
$$

With the same arguments, we can deduce the relationship between the normal load and the normal stress:

$$
\sigma=\frac{\phi k}{4 \pi R^{2}} F \sim \frac{F}{R^{2}}
$$

Assuming a general relationship of the form of Eq. 3] one gets therefore an adhesive stress which depends on the normal stress as shown in Fig. 2

$$
c_{\mathrm{adh}}=c_{0}\left(\frac{\sigma}{\sigma_{0}}\right)^{n}
$$

where $n, \sigma_{0}$ and $c_{0}$ characterize the properties of the material. This relationship is our general starting point to study the stability of a cohesive heap.

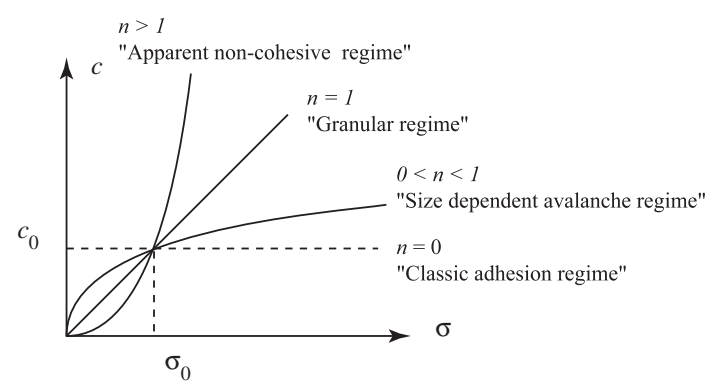

FIG. 2: different regimes of cohesion in a granular heap.

\section{FAILURE OF A COHESIVE HEAP}

The most simple approach in the continuum limit to study the failure of a granular material is the plastic theory of Mohr-Coulomb. The validity of the continuum approach has been widely discussed. Nevertheless continuum approaches allow to predict the Green's function of a granular layer 31] and is widely used in soil mechanics [17, 27]. We consider the stability of a cohesive granular heap, characterized by an adhesive stress depending on the normal stress, as given in Eq. [7 in which we suppose the Mohr-Coulomb criterion valid. Our aim is to locate in such a material the "slip plane", where failure occurs. The basis of our analysis is the Coulomb criterion: a granular material is stable if for each surface inside the material the following inequality is obeyed:

$$
\tau \leq \mu \sigma
$$

where $\tau$ is the shear stress and $\sigma$ is the normal stress. For a cohesive material, we assume that this condition can be generalized by adding in Eq. 8 the adhesive stress $c_{\text {adh }}$, as defined in the previous section, to the normal stress $\sigma$ 7].

We consider the geometry depicted in Fig. 3. A granular heap, with height $H$, makes an angle $\theta$ with the horizontal. The heap is supposed to be invariant in the direction perpendicular to the figure. We follow and generalize the approach given in Ref. 17] to the case of a cohesive material with the law of cohesion given in Eq. 7 In the simplified description of Ref. [17], the slip surface is assumed to be planar, making an angle $\alpha$ against the horizontal.

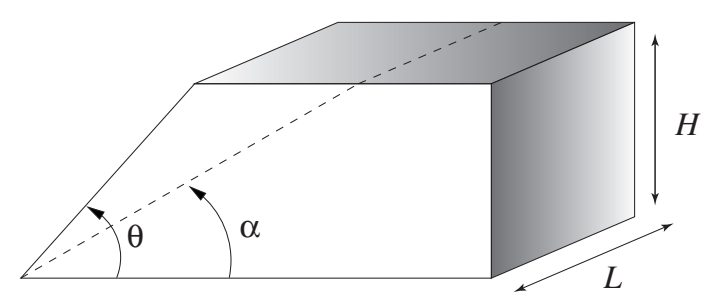

FIG. 3: Geometry of the granular heap. $\theta$ is the actual slope of the heap, while $\alpha$ locates the position of a possible failure.

The force balance in the direction perpendicular and parallel to the slip plane leads to the following conditions:

$$
F=P \sin \alpha \quad N=P \cos \alpha+F_{\text {adh }}
$$

where $P$ is the weight of the granular material above the slip plane; $c=F / S_{\text {slip }}$ and $\sigma+c_{\text {adh }}=N / S_{\text {slip }}$ are the normal and tangential stress along the possible slip plane

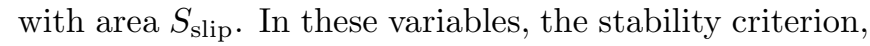
Eq. 8 writes $F<\mu N$. A simple geometric calculation 
leads to the relationships [17]:

$$
\begin{aligned}
P & =\frac{1}{2} \rho g L H^{2}\left(\frac{1}{\tan \alpha}-\frac{1}{\tan \theta}\right) \\
S_{\text {slip }} & =\frac{H L}{\sin \alpha}
\end{aligned}
$$

with $L$ the length of the heap in the invariant direction (perpendicular to the figure in Fig. 31). Gathering these results, one gets the following stability criterion:

$$
\frac{1}{2} \rho g H^{2}\left(\frac{1}{\tan \alpha}-\frac{1}{\tan \theta}\right)(\sin \alpha-\mu \cos \alpha) \leq \mu \frac{c_{\text {adh }} H}{\sin \alpha}
$$

Introducing the angle $\theta_{0}$ defined as $\tan \theta_{0}=\mu$, this relation can be adequately rewritten as:

$$
\sin (\theta-\alpha) \sin \left(\alpha-\theta_{0}\right) \leq 2 \sin \theta_{0} \sin \theta \frac{c_{\text {adh }}}{\rho g H}
$$

Now, one has to introduce the dependence of the cohesive stress $c_{\text {adh }}$ as a function of the normal stress, as discussed in the previous section. We shall use the general expression given by Eq. 7 Using $\sigma=P \cos \alpha / S_{\text {slip }}$, one gets after some algebra:

$$
c_{\mathrm{adh}}=c_{0}\left(\frac{\rho g H}{2 \sigma_{0}}\right)^{n}\left(\frac{\sin (\theta-\alpha) \cos \alpha}{\sin \theta}\right)^{n}
$$

Introducing the cohesion parameter $\zeta_{\text {coh }}$ defined as:

$$
\zeta_{\mathrm{coh}}=\frac{c_{0}}{\rho g H}\left(\frac{\rho g H}{2 \sigma_{0}}\right)^{n}
$$

and the function $f[\alpha]$ defined as:

$$
f[\alpha]=\frac{(\sin (\theta-\alpha))^{1-n}}{(\cos \alpha)^{n}} \sin \left(\alpha-\theta_{0}\right)
$$

Eq. 12 rewrites:

$$
f[\alpha] \leq \zeta_{\mathrm{coh}} \sin \theta_{0}(\sin \theta)^{n-1}
$$

The heap is therefore stable at an angle $\theta$ if this inequality is verified for all possible $\alpha$ (with $0 \leq \alpha \leq \theta$ ). In the opposite case when this inequality is not verified for some values of $\alpha$, the slip plane corresponds to the value of $\alpha$ for which this inequality is "first" violated. Note that for a non-cohesive material, $\zeta_{\mathrm{coh}}=0$, the maximum angle of stability is simply $\theta_{0}$.

\section{A. $n \leq 1$ case}

First if $\theta \leq \theta_{0}$, the function $f[\alpha]$ is always negative and the stability condition is trivially verified: For $\theta<\theta_{0}$ the heap is always stable.

The opposite case $\theta>\theta_{0}$ is more complex. A typical plot for this function is then given in figure 4 This

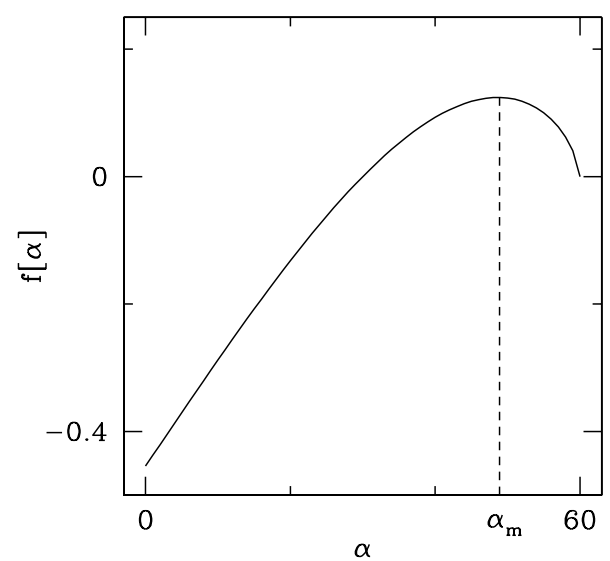

FIG. 4: Plot of $f[\alpha]$ as a function of $\alpha$, for a specific choice of parameters: $\theta=60^{\circ}, \theta_{0}=30^{\circ}$ and $n=1 / 3$. The dashed line locates the position of the maximum $\alpha_{\mathrm{m}}$.

function does exhibit a maximum for a value $\alpha_{\mathrm{m}}$, verifying $\partial f / \partial \alpha\left(\alpha_{\mathrm{m}}\right)=0$, and depending of the heap angle $\theta$. According to criterion [16] the heap is stable if $f\left[\alpha_{\mathrm{m}}\right] \leq \zeta_{\mathrm{coh}} \sin \theta_{0}(\sin \theta)^{n-1}$. The maximum angle of stability $\theta_{\mathrm{m}}$ thus is solution of the implicit equation:

$$
f\left[\alpha_{\mathrm{m}}\right]=\zeta_{\mathrm{coh}} \sin \theta_{0}\left(\sin \theta_{\mathrm{m}}\right)^{n-1} .
$$

A straightforward calculation shows that $\alpha_{\mathrm{m}}$ obeys the following relationship:

$\sin \left(2 \alpha_{\mathrm{m}}-\theta_{0}-\theta\right) \cos \alpha_{\mathrm{m}}=n \cos \left(2 \alpha_{\mathrm{m}}-\theta\right) \sin \left(\alpha_{\mathrm{m}}-\theta_{0}\right)$

It is interesting to consider the two limiting cases $n=1$ and $n=0$.

- for $n=1$, the solution of Eq. 18 is: $\alpha_{\mathrm{m}}=\theta$; and $\theta_{\mathrm{m}}$ is found to obey:

$$
\frac{\sin \left(\theta_{\mathrm{m}}-\theta_{0}\right)}{\cos \theta_{\mathrm{m}} \sin \theta_{0}}=\zeta_{\mathrm{coh}}
$$

This relationship can be rewritten in a more explicit form as :

$$
\tan \left(\theta_{\mathrm{m}}\right)=\mu\left(1+\zeta_{\mathrm{coh}}\right)=\mu\left(1+\frac{c_{0}}{2 \sigma_{0}}\right)
$$

with $\mu=\tan \theta_{0}$. This case presents two important characteristics: (i) failure occurs here at the surface of the heap, as emphasized by the relationship $\alpha_{\mathrm{m}}=\theta$; (ii) whatever the heap height $H$, there is always an effect of cohesion on the maximum angle of stability. This regime has been called the "granular regime" according to Tegzes experiments in which a cohesion effect on the avalanche angle is found without dependancy on the heap size. In Tegzes experiments, this regime is found at low liquid content. In section II we showed that the value 
$n=1$ is indeed expected when cohesion results of small capillary bridges between surfaces asperities.

- for $n=0$ ("classic adhesion regime" i.e. constant adhesion force), the solution of Eq. 18 is: $\alpha_{\mathrm{m}}=\left(\theta_{0}+\theta\right) / 2$, and $\theta_{\mathrm{m}}$ verifies the equation:

$$
\frac{1-\cos \left(\theta_{\mathrm{m}}-\theta_{0}\right)}{2 \sin \theta \sin \theta_{0}}=\zeta_{\mathrm{coh}}=\frac{c_{0}}{\rho g H}
$$

This is a classical result, as obtained e.g. in Ref. 17]. It is important to note that in this case, (i) the heap fails deep inside the material, since $\alpha_{\mathrm{m}}<\theta_{\mathrm{m}}$; (ii) the maximum angle of stability $\theta_{\mathrm{m}}$ depends on the height of the heap $H$. Such a dependence is in fact expected when one realizes that for $n=0$, a "capillary length scale" can be defined on dimensional grounds : $\ell_{\text {cap }}=c_{0} / \rho g$ (see eg Eq. 14). This capillary legnth gives the size of a macroscopic element of the granular medium whose weight equals the adhesion force which act on it. For instance it maybe the maximum size of a powder aggregat which can remain stuck under a horizontal surface. Eq. 21] shows that the maximum angle of stability of the heap reduces to $\theta_{0}$ when the size of the heap is very large compared to the "capillary length".

- For $n$ in between these two limiting cases, one has to solve numerically Eqs. [18 and [17 $\alpha_{\mathrm{m}}$ lies in between $\theta$ and $\left(\theta+\theta_{0}\right) / 2$. We show in Fig. 5 a typical result for the dependence of $\theta_{\mathrm{m}}$ and $\alpha_{\mathrm{m}}$ as a function of $\zeta_{\text {coh }}$ for $n=1 / 3$. The dependence of

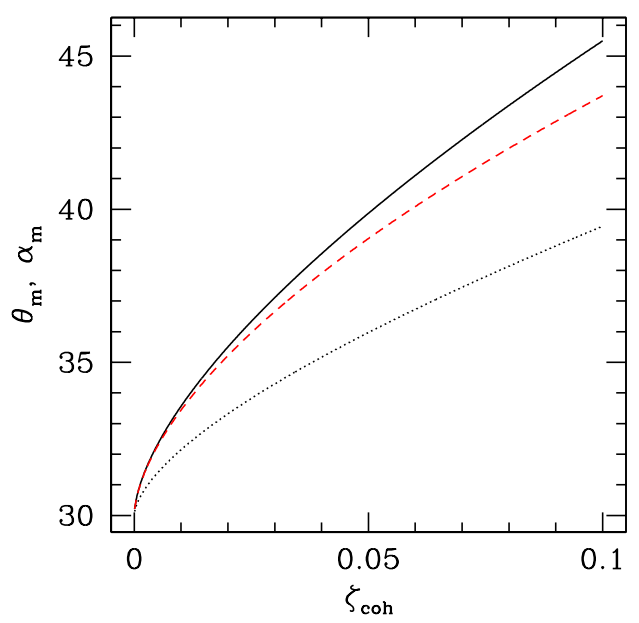

FIG. 5: Dependence of the maximum angle of stability, $\theta_{\mathrm{m}}$ (solid line) and failure plane location, $\alpha_{\mathrm{m}}$ (dotted line), as a function of the cohesion $\zeta_{\text {coh }}$ (angles are here given in degrees). Physical parameters are: $\theta_{0}=30^{\circ}, n=1 / 3$. The dashed line is the approximate solution, as given by Eq. 22

$\theta_{\mathrm{m}}$ in the limit of small cohesion can be computed analytically. For $\zeta_{\mathrm{coh}}=0$, one has $\alpha_{\mathrm{m}}=\theta_{\mathrm{m}}=\theta_{0}$ and one may expand the angles around this values for small $\zeta_{\mathrm{coh}}: \alpha_{\mathrm{m}}=\theta_{0}+\delta \alpha$ and $\theta_{\mathrm{m}}=\theta_{0}+\delta \theta$. First linearizing Eq. [18), one gets the relationship $\delta \alpha=\delta \theta /(2-n)$. Introducing this condition into Eq. [17), one gets eventually:

$$
\theta_{\mathrm{m}}-\theta_{0}=\gamma \zeta_{\mathrm{coh}}^{\frac{1}{2-n}}
$$

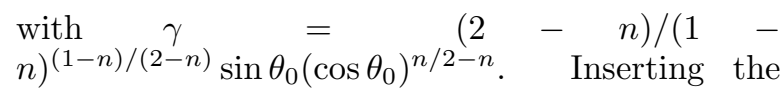
$H$ dependence of $\zeta_{\text {coh }}$, as defined in Eq. 14 one gets therefore

$$
\theta_{\mathrm{m}}-\theta_{0} \propto H^{\frac{n-1}{2-n}}
$$

This shows in particular that the dependence of the maximum angle on the heap height is a direct measure of the power $n$, characterizing the normal stress dependence of the cohesion stress. This regime is called the "size dependent avalanche" regime.

\section{B. $n>1$ case}

In this case, it is easy to show that whatever the cohesion, the maximum angle of stability is $\theta_{0}$. This regime is called the "apparent non-cohesive" regime. First as in the previous case, when $\theta<\theta_{0}$, the condition of stability, Eq. [16), is trivially obeyed since the function $f[\alpha]$ is negative. Now, for $\theta>\theta_{0}, f[\alpha]$ goes to infinity when $\alpha \rightarrow \theta$. The heap is therefore always unstable, whatever the cohesion. As a result, $\theta=\theta_{0}$ is the maximum angle of stability, independently of the cohesion $\zeta_{\text {coh }}$

\section{DISCUSSION}

In this paper we have studied the effect of an adhesive stress on the localization of the slip plane in an avalanche process. More precisely, we have shown that in a lot of practical case, the adhesive stress in a material depends on the normal stress. We have calculated, using a MohrCoulomb analysis, the internal angle of slip $\alpha_{\mathrm{m}}$.

A few conclusions can be drawn from these results:

- First, the location of the slip plane, here defined through $\alpha_{\mathrm{m}}$, does also strongly depend on this functional dependence: While for a constant cohesion (i.e. independent of the normal stress) the heap slips deep inside, only surface slip is expected when cohesion is linearly related to normal stress.

- Second the maximum static angle, $\theta_{\mathrm{m}}$, does depend on cohesion via the dimensionless parameter, $\zeta_{\text {coh }}$, defined as $\zeta_{\mathrm{coh}}=\frac{c_{0}}{\rho g H}\left(\frac{\rho g H}{2 \sigma_{0}}\right)^{n}$. An important remark is that for $n \neq 1$, the maximum static angle depends on the height of the heap, $H$. On the other hand, for the specific value $n=1$ - which 
is expected in some physical situations (see discussion in section [1A)- this dependence disappears and $\theta_{\mathrm{m}}$ is an intrinsic property of the material, independently of the geometry.

- Eventually, a change of regime in the cohesion, e.g. a change from $n=1$ to $n=0$ as discussed in section 【A will not only modify the dependence of $\theta_{\mathrm{m}}$ on cohesion, but more dramatically, it will change the localization of the slip plane: For example, while for $n=1$ slip occurs at the surface of the the heap $\left(\alpha_{\mathrm{m}}=\theta_{\mathrm{m}}\right)$, it will fracture in the interior of the heap for $n=0\left(\alpha_{\mathrm{m}}<\theta_{\mathrm{m}}\right)$. In other words, any change of slip behaviour reflects a transition of cohesion regime.

This features provide a framework to explain the results of Tegzes et al.. In view of the previous results, it seems natural to interprete the different regimes observed in the experiment, as an indication of a change in the cohesion regime. At low liquid content, the avalanche takes place at the surface of the heap and the angle increases linearly with the adhesion. With our approach, this would correspond to the $n=1$ regime, which is indeed expected at low liquid content (where the capillary bridges do not fill the full interstitial space between the grains). At higher liquid content, a change to a regime with $n<1$ is expected. This might explain the various observed experimental features, such as the size dependence.

As a conclusion, we hope that this work will motivate further experimental investigation on the stability of cohesive granular materials. The present results suggest that a careful determination of the stability properties and of the failure plane localization yields information on the cohesion forces between grains. In particular the heap height dependence of the maximum angle of stability should provide a direct measure of the cohesion properties. Another interesting geometry is the cylindrical bunker ("Janssen's problem"), in which cohesion effects, as discussed here, should play a particularly important role.

\section{Acknowledgments}

We thank the Région Rhône-Alpes for her financial support (Programme EMERGENCE 021892601).
[1] H. M. Jaeger, C.-H. Liu, and S. Nagel, Phys. Rev. Lett. 62, 40 (1988).

[2] J. Rajchenbach, Phys. Rev. Lett. 65, 2221 (1990).

[3] H. Jaeger and S. R. Nagel, Science 255, 1523 (1992).

[4] R. Albert, I. Albert, D. Hornbaker, P. Schiffer, and A. L. Barabási, Phys. Rev. E 56, R6271 (1997).

[5] R. Albert, M. Pfeifer, A. L. Barabási, and P. Schiffer, Phys. Rev. Lett. 82, 205 (1999).

[6] D. Hornbaker, R. Albert, I. Albert, A.-L. Barabási, and P. Shiffer, Nature 387, 765 (1997).

[7] L. Bocquet, E. Charlaix, S. Ciliberto, and J. Crassous, Nature 396, 735 (1998).

[8] C. Ursini, F. Restagno, G. Gayvallet, and E. Charlaix, Phys. Rev. E 66, 021304 (2002).

[9] N. Fraysse, H. Thomé, and L. Petit, Eur. Phys. J. B 11, 615 (1999).

[10] M. Valverde, A. Castellanos, and A. Ramos, Phys. Rev. E 62, 6851 (2000).

[11] M. Quintanilla, A. Castellanos, J. Valverde, Phys. Rev. E 64, 031301 (2001).

[12] P. Tegzes, R. Albert, M. Paskvan, A. L. Barabási, T. Vicsek, and P. Schiffer, Phys. Rev. E 60, 5823 (1999).

[13] A. J. Forsyth, S. R. Hutton, M. J. Rhodes, and C. F. Osborne, Phys. Rev. E 63, 031302 (2001).

[14] N. Olivi-Tran, N. Fraysse, P. Girard, M. Ramonda, and D. Chatain, Eur. Phys. J. E 25, 217 (2002).

[15] S. T. Nase, W. L. Vargas, A. A. Abatan, and J. J. Mccarthy, Powder Technol 116, 214 (2001).

[16] T. Mikami, H. Kamiya, and M. Horio, Chem. Eng Sci. 53, 1927 (1998).

[17] R. Nedderman, Statics and kinematics of granular materials (Cambridge University Press, Cambridge, 1992).

[18] K. L. Johnson, K. Kendall, and A. Roberts, Proc. Roy. Soc. London A 324, 301 (1971).
[19] B. Derjaguin, V. Muller, and Y. Toporov, Colloids Surf. 7, 251 (1983).

[20] D. Maugis, Journal of Colloid and Interface Science 150, 243 (1992).

[21] J. Israelachvili, Intermolecular \& Surface Forces (Academic Press, London, 1992), 2nd ed.

[22] F. Restagno, J. Crassous, C. Cottin-Bizonne, and E. Charlaix, Phys. Rev. E 65, 042301 (2002).

[23] F. Bowden and D. Tabor, The Friction and Lubrification of Solids (Clarendon Press, Oxford, 1950).

[24] J. Greenwood, J. Lubric. Tech. Trans. ASME 1, 81 (1967).

[25] L. D. Landau and E. M. Lifchitz, Theory of elasticity (Butterworth-Heinemann, New-York, 1986).

[26] The force-stress relationship could be in principle affected by the existence of the so-called force networks, which have been exhibited in various experiments and simulations in non cohesive systems [28, 29] and in slightly cohesive systems 30]. However, although this problem is still under debate in the litterature, macroscopic results have not been found to be incompatible with continuum approaches. We shall thus stay, in a first approximation, at such a continuum level.

[27] T. Mason, A. Levine, D. Ertas, and T. Halsey, Phys. Rev. E 60, R5044 (1999).

[28] F. Radjai, M. Jean, J. J. Moreau, and et al., Phys. Rev. Lett. 77, 274 (1996).

[29] P. Claudin, J. P. Bouchaud, M. E. Cates, and J. P. Wittmer, Phys. Rev. E 57, 4441 (1998).

[30] S. J. Antony, Phys. Rev. E 63, 011302 (2001).

[31] G. Reydellet and E. Clement, Phys. Rev. Lett. 86, 3308 (2001). 